\title{
Dopaminergic Effect on Non-Motor Symptoms in Late Stage Parkinson's Disease
}

Kristina Rosqvist, Per Odin, Peter Hagell, Susanne Iwarsson, Maria H. Nilsson, and Alexander Storch

[Journal of Parkinson's Disease 8(3), 2018, 409-420. DOI 10.3233/JPD-181380]

https://content.iospress.com/articles/journal-of-parkinsons-disease/jpd181380

On page 415, in Table 3, there is a typo. In the column vertical 'D6: Gastrointestinal', horizontal 'NMSS Score in "On" state':

The numbers given are incorrectly: 12.0 (4.0-20.0).

The correct numbers are: 6.0 (2.7-12.0).

Below find the corrected version of Table 3:

Table 3

Non-Motor Symptoms in "off" and "on" states during L-dopa test as assessed with the modified NMSS $(\mathrm{N}=30)$

\begin{tabular}{|c|c|c|c|c|c|c|}
\hline \multirow[t]{2}{*}{ NMSS, item, domain } & \multicolumn{2}{|l|}{ "Off" state } & \multicolumn{2}{|l|}{ "On" state } & \multicolumn{2}{|l|}{ Difference* } \\
\hline & $\begin{array}{c}\text { Number of pts } \\
\text { with symptom } \\
\text { present, } \\
\mathrm{n}(\%)\end{array}$ & $\begin{array}{c}\text { NMSS score } \\
\text { (median, } \mathrm{q} 1-\mathrm{q} 3)\end{array}$ & $\begin{array}{l}\text { Number of pts } \\
\text { with symptom } \\
\text { present, } \\
\text { n }(\%)\end{array}$ & $\begin{array}{c}\text { NMSS score } \\
\text { (median, q1-q3) }\end{array}$ & $P$-Value & $\begin{array}{c}\text { Number of pts } \\
\text { with NMSS } \\
>\text { NMSS }_{\text {Onf }}, \\
n(\%)\end{array}$ \\
\hline 1. Light headedness & $14(47 \%)$ & $0.0(0.0-4.5)$ & $12(40 \%)$ & $0.0(0.0-4.0)$ & 0.516 & $4(13 \%)$ \\
\hline D1: Cardiovascular & $14(47 \%)$ & $0.0(0.0-4.5)$ & $12(40 \%)$ & $0.0(0.0-4.0)$ & 0.516 & $4(13 \%)$ \\
\hline 3. Daytime sleepiness & $19(63 \%)$ & $3.0(0.0-6.0)$ & $19(63 \%)$ & $3.0(0.0-6.0)$ & 0.125 & $5(17 \%)$ \\
\hline 4. Fatigue & $22(73 \%)$ & $3.5(0.0-8.0)$ & $20(67 \%)$ & $2.5(0.0-6.5)$ & 0.017 & $11(37 \%)$ \\
\hline 5. Difficulty falling asleep & $9(30 \%)$ & $0.0(0.0-3.3)$ & $8(27 \%)$ & $0.0(0.0-2.3)$ & 0.125 & $4(13 \%)$ \\
\hline 6. Restless legs & $16(53 \%)$ & $1.0(0.0-4.5)$ & $14(47 \%)$ & $0.5(0.0-4.0)$ & 0.406 & $5(17 \%)$ \\
\hline D2: Sleep/fatigue & $30(100 \%)$ & $10.0(6.0-18.3)$ & $28(93 \%)$ & $8.0(5.5-14.0)$ & 0.003 & $15(59 \%)$ \\
\hline 7. Lost interest in surrounding & $15(50 \%)$ & $0.5(0.0-6.0)$ & $14(47 \%)$ & $0.0(0.0-4.0)$ & 0.086 & $7(23 \%)$ \\
\hline 8. Lack motivation & $20(67 \%)$ & $3.5(0.0-9.0)$ & $20(67 \%)$ & $2.5(0.0-8.0)$ & 0.148 & $6(20 \%)$ \\
\hline 9. Feel nervous & $11(37 \%)$ & $0.0(0.0-4.5)$ & $9(30 \%)$ & $0.0(0.0-4.0)$ & 0.008 & $8(27 \%)$ \\
\hline 10. Seem sad & $19(63 \%)$ & $2.0(0.0-6.0)$ & $16(53 \%)$ & $2.0(0.0-4.5)$ & 0.037 & $9(30 \%)$ \\
\hline 11. Flat moods & $24(80 \%)$ & $4.0(1.0-6.0)$ & $19(63 \%)$ & $3.0(0.0-4.0)$ & 0.003 & $15(50 \%)$ \\
\hline 12. Difficulty experiencing pleasure & $15(50 \%)$ & $0.5(0.0-4.5)$ & $13(43 \%)$ & $0.0(0.0-4.0)$ & 0.007 & $10(33 \%)$ \\
\hline D3: Mood/apathy & $27(90 \%)$ & $14.0(6.0-38.3)$ & $25(83 \%)$ & $13.0(4.0-26.3)$ & $<0.001$ & $21(70 \%)$ \\
\hline 16. Concentration & $20(67 \%)$ & $2.0(0.0-6.0)$ & $16(53 \%)$ & $1.0(0.0-4.0)$ & 0.006 & $9(30 \%)$ \\
\hline 17. Forget things or events & $21(70 \%)$ & $3.0(0.0-6.0)$ & $22(73 \%)$ & $2.0(0.0-4.0)$ & 0.078 & $10(33 \%)$ \\
\hline 18. Forget to do things & $19(63 \%)$ & $2.5(0.0-6.0)$ & $16(53 \%)$ & $1.0(0.0-4.5)$ & 0.232 & $8(27 \%)$ \\
\hline D5: Attention/memory & $28(93 \%)$ & $10.0(2.0-16.5)$ & $28(93 \%)$ & $6.0(2.0-11.0)$ & 0.006 & $15(50 \%)$ \\
\hline 19. Saliva & $23(77 \%)$ & $4.0(1.5-9.0)$ & $22(73 \%)$ & $4.0(0.0-9.0)$ & 0.359 & $6(20 \%)$ \\
\hline 20. Swallowing & $14(47 \%)$ & $0.0(0.0-6.0)$ & $14(47 \%)$ & $0.0(0.0-4.0)$ & 0.031 & $6(20 \%)$ \\
\hline
\end{tabular}


Table 3

(Continued)

\begin{tabular}{|c|c|c|c|c|c|c|}
\hline NMSS, item, domain & "Off" state & & "On" state & & Difference* & \\
\hline & $\begin{array}{c}\text { Number of pts } \\
\text { with symptom } \\
\text { present, } \\
\mathrm{n}(\%)\end{array}$ & $\begin{array}{c}\text { NMSS score } \\
\text { (median, q1-q3) }\end{array}$ & $\begin{array}{l}\text { Number of pts } \\
\text { with symptom } \\
\text { present, } \\
\mathrm{n}(\%)\end{array}$ & $\begin{array}{c}\text { NMSS score } \\
\text { (median, q1-q3) }\end{array}$ & $P$-Value & $\begin{array}{c}\text { Number of pts } \\
\text { with NMSS } \\
>\text { NMSS }_{\text {Off }}, \\
n(\%)\end{array}$ \\
\hline D6: Gastrointestinal & $28(93 \%)$ & $8.0(4.0-12.0)$ & $27(90 \%)$ & $6.0(2.7-12.0)$ & 0.009 & $12(40 \%)$ \\
\hline 22. Urgency & $22(73 \%)$ & $5.0(0.0-12.0)$ & $19(63 \%)$ & $4.0(0.0-12.0)$ & 0.031 & $6(20 \%)$ \\
\hline 23. Frequency & $18(60 \%)$ & $4.0(0.0-12.0)$ & $17(57 \%)$ & $3.0(0.0-12.0)$ & 0.500 & $5(17 \%)$ \\
\hline 24. Nocturia & $24(80 \%)$ & $8.0(2.0-12.0)$ & $24(80 \%)$ & $5.0(1.8-12.0)$ & 0.063 & $5(17 \%)$ \\
\hline D7: Urinary & $27(90 \%)$ & $15.0(4.0-33.8)$ & $26(87 \%)$ & $13.0(4.0-29.3)$ & 0.004 & $9(30 \%)$ \\
\hline 25. Interest in sex & $6(20 \%)$ & $0.0(0-0.0)$ & $6(20 \%)$ & $0.0(0.0-0.0)$ & 1.000 & $0(0 \%)$ \\
\hline 26. Problems having sex & $15(50 \%)$ & $4.0(0.0-12.0)$ & $15(50 \%)$ & $4.0(0.0-12.0)$ & 1.000 & $0(0 \%)$ \\
\hline D8: Sexual dysfunction & $16(53 \%)$ & $10.0(0.0-12.0)$ & $16(53 \%)$ & $10.0(0.0-12.0)$ & 1.000 & $0(0 \%)$ \\
\hline 27. Pain & $15(50 \%)$ & $0.5(0.0-8.0)$ & $15(50 \%)$ & $0.5(0.0-6.5)$ & 0.766 & $4(13 \%)$ \\
\hline 30. Excessive sweating & $8(27 \%)$ & $0.0(0.0-1.0)$ & $5(17 \%)$ & $0.0(0.0-0.0)$ & 0.188 & $5(17 \%)$ \\
\hline D9: Miscellaneous & $19(63 \%)$ & $3.0(0.0-8.0)$ & $18(60 \%)$ & $2.5(0.0-7.3)$ & 0.559 & $5(17 \%)$ \\
\hline NMSS total score & $30(100 \%)$ & $79.0(47.8-115.3)$ & $30(100 \%)$ & $63.5(44.8-96.3)$ & $<0.001$ & $21(70 \%)$ \\
\hline
\end{tabular}

NMSS, Non-Motor Symptoms Scale (modified; 0-276, higher = worse); q1-q3, first and third quartiles; D, domain; Pts, patients. Symptom present $=$ score $\geq 1$ on each item/domain. NMSS score $=$ severity $\times$ frequency. *Differences between defined "off" and "on" states; number of patients who reported worse symptoms in the "off" than in the "on" state. $P$-values are from Wilcoxon sign-rank test for the comparisons of NMSS score between the defined "off" and "on" states. $P$-values were not adjusted for multiple testing. Bold $P$-values are statistically significant at $P<0.05$. 